\title{
Electronegative LDL: Could it be a Potential Biomarker for Organ Interactions?
}

\author{
Huseyin Vural ${ }^{1^{*}}$, Suheyla Akyol ${ }^{2}$
}

${ }^{1}$ RWTH University, Institute of Molecular Pathobiochemistry, Experimental Gene Therapy and Clinical Chemistry, Aachen, Germany

${ }^{2}$ Suleyman Demirel University, School of Medicine, Isparta, Turkey

\section{Correspondence:}

Huseyin Vural

Address: RWTH University, Institute of Molecular Pathobiochemistry, Experimental Gene Therapy and Clinical Chemistry, Aachen, Germany

Email: drvural@gmail.com
Received: 02.08.2021, Accepted: 21.09.2021

\begin{abstract}
Obstructive Sleep Apnoea Syndrome (OSAS) and obesity are linked by a bidirectional causality, where the effects of one affect the other. The factors most involved in the association between OSAS and obesity are oxidative stress, systemic inflammation, and gut microbiota. Moreover, there is a potential link between reduced lung function due to sharing common or similar inflammatory processes and the risk of non-alcoholic fatty liver disease (NAFLD). Therefore, we hypothesize that decreased lung function could be a contributory factor to the development of NAFLD. Electronegative LDL (L5), a promising clinical biomarker for cardiovascular disease, has an important role in (cardiovascular disease) CVD risk assessment. So, L5 may be a potential biomarker for such dual organ interactions and related diseases, too.
\end{abstract}

Keywords: OSAS, obesity, NAFLD, electronegative LDL

The review article entitled 'The relationship between obstructive sleep apnoea syndrome and obesity: A new perspective on the pathogenesis in terms of organ crosstalk' by Kuvat et al. provides current information concerning the organ interactions and relation potential health effects [1]. Due to the interaction of organs, functional failure of one organ can lead to the dysfunction of others. Adipose tissue as a main role player of obesity, liver, and intestines have been suggested to play essential roles in organ interactions related to mechanisms involved in the pathogenesis of OSAS [1,2].

It is reported that obesity could result in OSAS via several mechanism including the increased collapsibility of the pharyngeal airway through excessive fat deposition [3] as well as oxidative stress and inflammation [1]. Also, the relationship between lung function and cardio-metabolic conditions has also been highlighted. A previous study has demonstrated that decreased lung function is associated with an increase in low-grade inflammation and increased risk of diabetes, cardiovascular disease, and metabolic syndrome [4].
After all this, in a recent research article, it is reported that reduced lung function was a risk factor for incident NAFLD [5]. It is also suggested a pathophysiological links among metabolic syndrome, NAFLD and chronic obstructive pulmonary disease [6]. Indeed, OSAS has been associated with all the components of metabolic syndrome, including NAFLD [7]. Increasing evidence now acknowledges the frequent association between OSAS and metabolic syndrome as well as between OSAS and NAFLD $[8,9]$. On the other hand, it is claimed that pulmonary function is being deteriorated with worsening histological severity of NAFLD, especially at the fibrosis stage [10].

Electronegative LDL (L5), a form of modified LDL, can be detected in different amount ranges in both healthy individuals and patients with various diseases [11]. Since L5 may be a promising biomarker, some authors have emphasized its association with multiple cardiovascular disease risk factors and CVD risk, suggesting that the LDL electronegativity index may have the potential to be a novel index for predicting CVD [12,13].

It is revealed that adiponectin to be beneficial effects in maintaining vascular 
homeostasis [14]. A recent study's findings provided novel insight into the antiatherogenic mechanisms of adiponectin. In this study, Kakino et al. [15] reported that adiponectin not only binds to atherogenic LDL and inactivates it, but also prevents it from entering the cell, thus showing an antiatherogenic effect. Interestingly, it was noticed adiponectin was abundant in L5 but not in L1, the least electronegative subfraction of LDL.

In conclusion, both OSAS and NAFLD are among the risk factors for CVD, it is remarkable that these chronic diseases are associated with obesity and have similar organ interactions. Due to pathological processes such as endothelial dysfunction, inflammation, and oxidative stress, L5 may be a potential biomarker in such chronic and progressive diseases [16]. The current data suggest that L5 levels may represent a new diagnostic and therapeutic target in chronic and progressive diseases [17]. Therefore, future studies should focus on the complex interaction between these pathways, and more comprehensive and well sampled clinical studies on the L5 biomarker should be conducted.

Author contributions: All authors have sufficiently contributed to the study, and agreed with the results and conclusions.

Funding: No funding source is reported for this study.

Declaration of interest: No conflict of interest is declared by authors.

\section{REFERENCES}

1. Kuvat N, Tanriverdi H, Armutcu F. The relationship between obstructive sleep apnea syndrome and obesity: A new perspective on the pathogenesis in terms of organ crosstalk. Clin Respir J 2020; 14: 595-604. doi: 10.1111/crj.13175.

2. Armutcu F. Organ crosstalk: the potent roles of inflammation and fibrotic changes in the course of organ interactions. Inflamm Res. 2019; 68(10): 825-39. doi: 10.1007/s00011-019-01271-7.

3. Isono $\mathrm{S}$. Obesity and obstructive sleep apnoea: mechanisms for increased collapsibility of the passive pharyngeal airway. Respirology. 2012; 17(1): 32-42. doi: 10.1111/j.1440-1843.2011.02093.x.

4. Kwon CH, Rhee EJ, Song JU et al. Reduced lung function is independently associated with increased risk of type 2 diabetes in Korean men. Cardiovasc Diabetol. 2012; 11: 38. doi: 10.1186/1475-2840-11-38.

5. Song JU, Jang Y, Lim SY, et al. Decreased lung function is associated with risk of developing non-alcoholic fatty liver disease: A longitudinal cohort study. PLoS One. 2019; 14(1): e0208736. doi: 10.1371/journal.pone.0208736
6. Katsiki N, Stoian AP, Steiropoulos P, Papanas N, Suceveanu AI, Mikhailidis DP. Metabolic Syndrome and Abnormal Peri-Organ or Intra-Organ Fat (APIFat) Deposition in Chronic Obstructive Pulmonary Disease: An Overview. Metabolites 2020; 10(11): 465. doi: 10.3390/metabo10110465

7. $\mathrm{Xu} \mathrm{S}$, Wan $\mathrm{Y}, \mathrm{Xu} \mathrm{M}$, et al. The association between obstructive sleep apnea and metabolic syndrome: A systematic review and meta-analysis. BMC Pulm. Med. 2015; 15: 105. doi: 10.1186/s12890-015-0102-3.

8. Musso G, Cassader M, Olivetti C, Rosina F, Carbone G, Gambino R. Association of obstructive sleep apnoea with the presence and severity of non-alcoholic fatty liver disease. A systematic review and meta-analysis. Obes. Rev. 2013; 14: 417-31. doi: 10.1111/obr.12020.

9. Jin S, Jiang S, Hu A. Association between obstructive sleep apnea and non-alcoholic fatty liver disease: A systematic review and meta-analysis. Sleep Breath. 2018; 22: 841-51. doi: 10.1007/s11325-018-1625-7.

10. Lee HW, Lee DH, Lee JK, et al. Pulmonary function is associated with fibrosis severity in patients with biopsyproven nonalcoholic fatty liver disease. Liver Int. 2020 Dec; 40(12): 3008-17. doi: 10.1111/liv.14626.

11. Akyol S, Lu J, Akyol O, et al. The role of electronegative low-density lipoprotein in cardiovascular diseases and its therapeutic implications. Trends Cardiovasc Med. 2017; 27(4): 239-46. doi: 10.1016/j.tcm.2016.11.002.

12. Hsu JF, Chou TC, Lu J, et al. Low-density lipoprotein electronegativity is a novel cardiometabolic risk factor. PLoS One. 2014; 9(9): e107340. doi: 10.1371/journal.pone.0107340.

13. Chang CT, Wang GJ, Kuo CC, et al. Electronegative Low-density Lipoprotein Increases Coronary Artery Disease Risk in Uremia Patients on Maintenance Hemodialysis. Medicine (Baltimore). 2016; 95(2): e2265. doi: 10.1097/MD.0000000000002265.

14. Sowka A, Dobrzyn P. Role of perivascular adipose tissuederived adiponectin in vascular homeostasis. Cells. 2021; 10(6): 1485. doi: 10.3390/cells10061485.

15. Kakino A, Fujita Y, Ke LY, Chan HC, Tsai MH, Dai CY, et al. Adiponectin forms a complex with atherogenic LDL and inhibits its downstream effects. J Lipid Res. 2020; 62: 100001. doi: 10.1194/jlr.RA120000767.

16. Armutcu F. Akyol S, Vural H. Metabolic syndrome is an important cornerstone in the health-disease line and pathological organ interaction. J Cell Signal. 2020; 1(3): 70-5. doi: 10.33696/Signaling.1.014.

17. Akyol O, Chowdhury I, Akyol HR, et al. Why are cardiovascular diseases more common among patients with severe mental illness? The potential involvement of electronegative low-density lipoprotein (LDL) L5. Medical Hypotheses 2020; 142: 109821. doi: 10.1016/j.mehy.2020.109821. 